\title{
Paraquat and Parkinson's disease
}

\author{
C Berry ${ }^{1}$, C La Vecchia ${ }^{2,3}$ and P Nicotera ${ }^{*, 4}$
}

As evidence emerges that complex gene alterations are involved in the onset of Parkinson's disease (PD), the role of environmental chemicals in the pathogenesis of this disease becomes intensely debated. Although it is undisputed that acute exposure to certain chemicals such as 1-methyl-4-phenyl-1,2,3,6-tetrahydropyridine (MPTP) is sufficient to cause human parkinsonism, the evidence that the risk for PD increases because of environmental exposure is generally weaker. Several studies have suggested that pesticide exposure and life in rural areas are significant risks factors for PD. Among other pesticides, paraquat $(P Q)$ has been linked to $P D$ by epidemiological studies and experimental work in rodents, in which it causes lesions in the substantia nigra, pars compacta. However, the evidence that human exposure to the chemical results in an increased risk for PD is rather limited and based on insufficient epidemiological data. This review critically analyses the evidence that implicates $P Q$ in parkinsonism and discusses the limitations of chemical modelling of PD.

Cell Death and Differentiation (2010) 17, 1115-1125; doi:10.1038/cdd.2009.217; published online 22 January 2010

Parkinsonism is a complex syndrome resulting from a number of different pathogeneses that give rise to various degrees of motor deficits, akinesia, rigidity, tremors and postural disturbance along with other neurological deficits, which manifest as the disease progresses. These are generally considered to be manifestations of toxicity in dopaminergic neurons as main target. Post-mortem analysis of the brains of Parkinson's disease (PD) patients has revealed the constant pathological finding that dopaminergic cell degeneration is most severe in the substantia nigra, pars compacta in which a consistent loss of $>60-70 \%$ of neurons is evident. Neuronal loss is less pronounced in other mid-brain regions. The mechanisms that make different neuronal subsets either resistant or more susceptible to parkinsonian degeneration are unknown as are the mechanisms responsible for neuronal injury and loss. It is generally agreed that the aetiology of PD is likely to involve both environmental and genetic factors. ${ }^{1,2}$ In recent years, several genes that participate in PD pathogenesis have been characterized. Alpha-synuclein and parkin are involved in familial PD, autosomal dominant and recessive PD, respectively. Their functions and that of other genes, including Omi/ HtrA2, PINK-1, DJ-1 and dardarin, has been the subject of intense studies and nested gene pathways are starting to be unveiled.

Nevertheless, there is widespread concern that epigenetic factors and in particular environmental chemicals can either cause PD or increase the risk of developing it. A number of toxins may produce parkinsonism. Exposure to 1-methyl-4phenyl-1,2,3,6-tetrahydropyridine (MPTP) has been shown to produce permanent parkinsonism in humans, non-human primates and rodents, by exerting an effect primarily on the function of mitochondrial complex I. Other known chemical triggers of parkinsonian syndromes include chronic exposure to high levels of manganese, which causes degeneration in the sub-thalamic nucleus and the pallidum and which improves after manganese chelation. Carbon disulphide $\left(\mathrm{CS}_{2}\right)$ can also cause neurobehavioural problems associated with a parkinsonian syndrome in relatively young subjects. However, both manganese and $\mathrm{CS}_{2}$ also cause other neurological problems and can hardly be considered specific dopaminergic toxins. The pesticide dieldrin has been found in post-mortem brain samples from individuals with PD and can exacerbate MPTP neurotoxicity in mice after perinatal exposure.

Paraquat $(P Q)$, a widely used pesticide, undergoes oneelectron reduction chemistry in cells that can sequester it, causing a widespread oxidative/nitrosative stress. In humans, ingestion of $P Q$ accidentally or for suicidal purposes causes severe pulmonary lesions, which are often fatal. To date, there is no evidence that acute exposure to $P Q$ causes brain lesions directly. On the other hand, PQ can induce PD-like lesions in certain mouse strains and rats. The underlying mechanisms are directly or indirectly related to reactive oxygen species. Because $P Q$ is used widely in agriculture, concern has been raised that exposure to this agent may increase the risk of developing $P D$ in the human population. In the following sections we will analyse the available epidemiological evidence as well as the leading hypothesis on the mechanism by which $P Q$ can affect neurons.

\footnotetext{
${ }^{1}$ Queen Mary, University of London, London, E1 4NS, UK; ${ }^{2}$ Istituto di Richerche Farmacologiche'Mario Negri', Via La Masa 19, 20156 Milan, Italy; ${ }^{3}$ Istituto di Statistica Medica e Biometria, University of Milan, 20133, Milan, Italy and ${ }^{4}$ Deutsche Zentrum für Neurodegenerative Erkrankungen (DZNE) Ludwig-Erhard-Alle 2, 53175 Bonn, Germany

*Corresponding author: P Nicotera, Deutsche Zentrum für Neurodegenerative Erkrankungen (DZNE) Ludwig-Erhard-Alle 2, 53175 Bonn, Germany. Tel: 4922830899 100; E-mail: Pierluigi Nicotera@dzne.de

Keywords: neurodegeneration; pesticides; parkinsonism; neurons; microglia

Abbreviations: PD, Parkinson's disease; MPTP, 1-methyl-4-phenyl-1,2,3,6-tetrahydropyridine; PQ, paraquat; $\mathrm{CS}_{2}$, carbon disulphide; UPDRS, united Parkinson's disease rating scale

Received 24.11.09; revised 03.12.09; accepted 07.12.09; Edited by R Knight; published online 22.1.10
} 


\section{Epidemiological Studies}

General considerations. Data relating to the possible association between pesticides, herbicides and PD are derived from case reports, ecological studies (e.g., analysing exposure through water levels of pesticides), mortality studies (analysing death certification in a defined area), case-control and cohort studies - with the last two being of greater value if inferences about aetiology are to be drawn.

In any epidemiological study in this area it is important to consider potential bias and confounding factors. For casecontrol studies both case ascertainment and control selection are possible sources of bias. Cases may be identified from hospitals or clinics, residential care homes or prescribing records for drugs of relevance. Hospital cases may be more severe (with the inference that less severe cases may be excluded). Controls may be population or hospital based, may include spouses of cases, subjects suggested by index cases and from families and friends. Friend and family controls may have similar exposures through a shared background. Recall bias is another major issue, particularly for neurodegenerative diseases. For cohort studies, definition of PD diagnosis, exposure ascertainment and the choice of the non-exposed comparison groups are key factors. Diagnostic criteria are another potentially important source of bias, as reviewed by Litvan et al. ${ }^{3}$ Potential confounding factors include age, wellwater consumption, farming, rural living, head trauma, a family history of PD, smoking and caffeine intake, and infectious diseases such as Nocardiosis, as all of these have been suggested to affect the incidence of PD.

Most studies have the major problem that there is no satisfactory information about definition, duration and extent of exposure. In addition, there is often no information about the specific types of pesticide used, or about those formulations available on the relevant market at the time when exposure took place. This also makes pooling of studies problematic.

Pesticides and PD. More than 40 epidemiological studies, published during the past three decades, provide data on the issue of pesticide exposure and the risk of PD. It seems that many of these studies were instigated after the suggestions made by Barbaeu $(1984)^{4}$ who, in a review paper that outlined a research strategy for PD, discussed the pathogenesis of parkinsonism induced by manganese intoxication and intoxication with MPTP. He wrote: 'although it is unlikely, except in drug addicts and chemists directly exposed, that Parkinson's disease is generally caused by MPTP, it is certainly possible that many people are constantly being exposed to products with a similar conformation found frequently in nature or industrially. Many herbicides, for example, including the well-known Paraquat, may have a similar chemical mode of action. In susceptible individuals such products could initiate the process of cell degeneration found in Parkinson's disease. This field deserves thorough scrutiny'. These epidemiological studies have been reviewed recently. ${ }^{5,6}$ Overall, about two-thirds of the studies gave relative risks (RR) above unity and one-third around or below unity, in the presence of a substantial heterogeneity.
Engel et al. ${ }^{7}$ considered exposures of 310 orchardists who reported parkinsonism and who had previously participated in a study of men occupationally exposed to pesticides. Structured neurological examination was undertaken, and the subjects filled in a questionnaire about their pesticide exposures. The mean age was 69.6 years; 238 were exposed to pesticides, and 72 were not. A nurse administered the united Parkinson's disease rating scale (UPDRS). No distinction was made between PD and parkinsonian-like illnesses. The conclusion was that parkinsonism might be associated with long-term use of pesticides, but not with any particular compound.

In a French cohort of elderly farmers, ${ }^{8}$ the RR for exposure to pesticides was $5.6(95 \% \mathrm{Cl} 1.5-21.6)$ in men (based on eight exposed cases), but no association was observed in women ( $R R=1.0,95 \% \mathrm{Cl} 0.2-4.8$ ). In a case-control study from Olmsted Country, Minnesota, ${ }^{9}$ based on 149 cases and 123 controls, the RR for any exposure to pesticides was 2.4 (95\% Cl 1.1-5.4).

The American Cancer Society Cancer Prevention Study II (ACS-CSS II) $^{10}$ was a large (143325) cohort study of lifestyle and cancer starting in 1992. This population was followed on a number of occasions, and in 2001 a question was asked about lifetime occurrence of PD. Participants were mostly white and the median age at enrolment was 63.7 years for men and 62 years for women. Occupational records were the source of exposure data and anyone recorded as 'farmers, ranchers or a fisherman' was considered to be a farmer. There were 840 responders who reported a diagnosis of PD. Questioning on exposure included asking about asbestos, gasoline, dyes, formaldehyde and wood dust. Only $15 \%$ reported pesticide exposure in the 'farming' group and a similar figure was reported for gasoline exhaust. Exposure to pesticides was reported by $5.7 \%$ of the total study population, and the corresponding RR was $1.7(95 \% \mathrm{Cl} 1.2-2.5)$ for non-farmers and $1.6(95 \% \mathrm{Cl} 0.9-2.7)$ for farmers. No specific pesticide was identified as a risk factor.

A multicentre study from five European countries ${ }^{11}$ found only a modest overall association between working in agriculture and PD (RR 1.13, 95\% Cl 0.83-1.57). Such an association may well be explained by bias. A case-control study of occupational factors in PD conducted in nine German clinics and including 390 cases and a total of 755 population controls $^{12}$ found RRs around 2 for professional herbicide or insecticide users, but only in one case was there a reported exposure to $\mathrm{PQ}$.

The issue of a possible association between $P Q$ and $P D$ received more attention after a study conducted in Taiwan between 1993 and 1995 on 120 patients with PD and 240 hospital controls was reported. ${ }^{13}$ Overall, 46 cases and 41 controls reported use of herbicides/pesticides, corresponding to a RR of 2.89 ( $95 \% \mathrm{Cl} 2.28-3.36)$. The RR rose to 6.72 (95\% $\mathrm{Cl}$ 2.62-17.21) for use for $\geq 20$ years. In all, 31 cases versus 21 controls reported paraquat use, corresponding to an RR of 3.22 (95\% Cl 2.41-4.31), which rose to 6.43 (95\% Cl $2.41-$ 17.2) for use for $\geq 20$ years. Information on use of herbicides and pesticides was self-reported, and not validated. Allowance for tobacco use and other possible confounding factors did not explain the association observed. The use of both PQ and other herbicides and pesticides conferred an apparently 
greater risk than the use of herbicides other than $P Q$. The researchers did not however compare exposure to $P Q$ alone versus other herbicides and against non-exposure.

In a study from urban and rural areas of Finland, including 123 cases of PD and 246 controls, ${ }^{14} 3$ cases versus 5 controls reported ever $P Q$ use, corresponding to a crude $R R$ of 1.2, non-significant. The RR for overall use of herbicides was 1.4 (95\% Cl 0.8-2.5), and that of pesticides was 1.0 (95\% 0.61.7). Thus, in that study also, the RR estimate for $P Q$ was not different from those of total herbicide and/or pesticide use.

A study from Washington State considered the proportional prevalence ratio (as a measure of $\mathrm{RR}$ ) of parkinsonism (rather than the full clinical picture of PD) according to exposure to selected herbicides. ${ }^{7}$ The RR for any exposure to pesticides was $0.8(95 \% \mathrm{Cl} 0.5-1.2)$ and that for exposure to $P Q$ was also $0.8(95 \% \mathrm{Cl} 0.5-1.3)$.

In a case-control study from the Group Health Cooperative in Washington State, ${ }^{15}$ including 156 cases of PD with information on occupational exposure, 19 cases versus 28 controls reported any exposure to pesticides, corresponding to a RR of $1.0(95 \% \mathrm{Cl} 0.5-1.9)$. Only two cases and two controls reported any $\mathrm{PQ}$ use $(\mathrm{RR}=1.7,95 \% \mathrm{Cl} 0.2-12.8)$.

The Agricultural Health Study ${ }^{16}$ made a cohort investigation of over 79500 agricultural workers from North Carolina and lowa. The participants were recruited between 1993 and 1997 and followed-up to 1999-2003. There were a total of 83 prevalent and 78 incident cases of PD. The researchers considered various exposures, including acute poisoning episodes and chronic exposures and took account of several confounding factors and the different farming practices of lowa and North Carolina. They found that the prevalence of neurological symptoms was associated with cumulative lifetime exposure to pesticides, notably organophosphates and organochlorines, and that this prevalence was mainly associated with chronic moderate exposure. Overall, 14 prevalent and 11 incident cases reported PQ use. The RRs, adjusted for all available confounding factors, were $1.8(95 \%$ Cl 1.0-3.4) for prevalent PD, and 1.0 (95\% Cl 0.5-1.9) for incident PD. These estimates were not heterogeneous when compared with those given for several other herbicides and pesticides. In fact, the overall RR of incident PD for the highest exposure level of all pesticides was $2.3(95 \% \mathrm{Cl} 1.2-4.5)$.

PQ-specific studies. Until recently at least seven studies have reported data on PQ use and PD. Of these, five are case-control studies, one is a cross-sectional study ${ }^{7}$ and one is a cohort investigation. ${ }^{16}$ However, the total number of PD cases reporting exposure to $P Q$ is only 95 .

Few data on exposure to paraquat are available from studies published in the 1990s from Canada and Germany. In particular, Hertzman et al. ${ }^{17}$ published two reports, in 1990 and 1994. The 1990 study was based on a case-control sample identified in the Kootenay region, a rural mountainous area of British Colombia with a reportedly high PD prevalence. Cases were identified by contacting physicians in the area who could identify patients with a diagnosis of PD who might respond to a postal questionnaire. The same neurologist subsequently examined all cases. Controls were randomly selected from regional electoral rolls and were of similar age and sex. Out of 57 cases of PD and 122 controls, 4 cases versus none in the control group reported ever handling $P Q$. The study had limited power due to the small sample size, and lacked details on risk factor exposure assessment. The 1994 study $^{18}$ included 127 cases and comparable numbers of controls: (1) randomly selected from electoral lists, and (2) cardiac disease patients in a rural area of British Colombia. Both quantity and frequency of exposure were assessed for a number of herbicides/pesticides. Chemicals were evaluated both singly and by categories and the analyses were run separately for men and women. No significant association between $P Q$ and $P D$ was consistently found across control groups or genders. The RR estimates were approximately 1.1-1.2 according to the type of comparison group (hospital/ community), and far from statistical significance.

The study from Taiwan, ${ }^{13}$ based on 120 PD cases (and 31 cases exposed to $P Q$ ), reported a significant excess risk for $P Q$, which nevertheless did not differ from that for overall herbicide or pesticide exposure. All the other studies reported nonsignificant RRs, close to unity or slightly above unity. Thus, the apparent association between herbicides (and PQ) and $P D$ in the study from Taiwan is likely to be due to some characteristics of the population or exposure, or due to some unrecognized bias or confounding. More importantly, there is no evidence that the relation between exposure to $P Q$ and $P D$ is different from that of other herbicides or pesticides, or - in the more general framework - occupation in agriculture as an indicator of PD risk.

With reference to review papers, Brown et al. ${ }^{6}$ identified 38 case-control studies including three abstracts that were included as they had been peer reviewed. In its conclusions, although commenting on the lack of power in most studies, the paper relied heavily on animal data to provide support for its view that there was evidence of an association of PD with herbicides and insecticides. This conclusion was reached despite the fact that the animal studies discussed were made at dose levels that had little to do with human exposure and were not by 'routes of administration that would be experienced by pesticide users'.

Li et al., ${ }^{5}$ in another review paper, concentrated on studies with good definition of PD, with some attempt at exposure assessment. They were critical of case studies. When 32 studies had been selected, they were 'scored' by an elaborate process with seven major criteria. With regard to $P Q$, the researchers considered that the studies of $\mathrm{Liou}^{19}$ were 'provocative', but pointed out that they have not been replicated. They considered that all of the studies had methodological flaws and concluded that there is no specific association of PD with herbicides, pesticides or any specific pesticide.

More recent studies have produced surprising associations. These have been ignored for reasons that are not always clear and that may be due to a failure to document other relevant exposures. Others, in attempts to overcome the problems of generalizations about exposure, have analysed evidence of selectively sensitive populations or particular exposures. Both approaches have difficulties. A study by Tanner et al. ${ }^{20}$ was constructed without overly restrictive diagnostic criteria as the researchers considered that this might exclude toxicantinduced cases. Thus, subjects with typical or atypical parkinsonism were included. The study was conducted in 
the United States between 2004 and 2007 with 519 PD cases and 511 paired controls. Of these, over $90 \%$ were assessed as having no exposure to pesticides. After adjustment for confounding factors, the data showed three significant occupational associations with parkinsonism: Legal Occupations (a threefold increased risk - although this is noted as consistent with three previous studies, it is dismissed as spurious), pesticide use (a twofold increased risk) and Construction and Extraction work (a 1.5-fold increased risk). Unlike the seven earlier studies quoted, this research was unable to detect an association between agricultural work and PD. Even the working in business and finance, legal occupations, construction and extraction, or transportation and material moving was associated with postural instability and gait difficulty subtype of parkinsonism.

The study acknowledges that there were no quantitative assessments of chemical exposure that 'recall bias' may affect the answers. They make the important point that in these case-control studies, causal relationships cannot be assumed. Attention is focussed on the associations between PD and an apparent occupational exposure to 2,4-D and the statistically nonsignificant associations between paraquat, permethrin and PD. These three chemicals have very small groups of 'exposed' cases, (16, 9 and 7, respectively) and even smaller matched control groups $(7,4$ and 2 , respectively). Because exposure to more than one of these pesticides was common, the researchers considered that future investigations should also assess the effects of combined exposure. They commented 'We found no association between the SOC code for agricultural work and parkinsonism, which confirms the suggestion that occupational code is a poor surrogate for pesticide use'. It is perhaps surprising that it is so often used in this way.

In another recent study, Costello et al. ${ }^{21,22}$ used a new approach for the assessment of pesticide exposure, incorporating California Pesticide Use Reporting (PUR) data and the geo-coding of residential addresses. The study considered only the use of the pesticides paraquat and maneb; information on exposure to other agricultural chemicals was not sought. Four time and age intervals were used in design and interpretation. They were firstly, an 'exposure period' of 19741999, for which PUR information was available. The information, available for each year, has been aggregated into two time 'windows', 1974-1989 and 1990-1999, but information has also been presented for the entire period. There was then a 'diagnosis period' from January 1998 to January 2007 and an 'enrolment period' from 1998 to 2007 . This seems to be the interval during which subjects were invited into the study and suggests that about one-half of the cases, but none of the controls, may have had previous contact with the researchers. It is interesting to note that results are provided for ages 35-60 and $>60$ years. Recruitment was helped by the cooperation of local neurologists, medical facilities, newspapers and radio stations. A total of 1167 cases were 'invited' to participate but of these only 563 were 'eligible'. In that, 96 eligible cases were excluded 'because they had other causes of Parkinsonism'. Of the 563 eligible cases, 65\% (368) provided all information needed for analyses. The reasons why the investigators excluded 395 cases, as their 'diagnosis date fell outside the 3-year range prior to contact', were not discussed. It is worth noting that if these cases were appropriately included in the study base they should belong to the denominator; this gives a participation rate of cases of $368 / 1165-(51+134)=37 \%$. It remains vague how controls were selected. About $45 \%$ of 'eligible' controls were included in the analyses but the response rate for all households approached is not provided; it seems to have been low. Notably, controls, but not cases, were selected from among property owners. The cases had a slightly higher percentage of males than did controls, had more first-degree relatives with PD, were substantially less educated but, nonetheless, smoked substantially less. The gradient with education was about twofold and as strong as that for cigarette smoking. More cases than controls had occupational exposures to pesticides and both the residential and occupational exposures probably included agents in addition to $P Q$ and maneb. The exposure information gathered for this study is very detailed. The pounds-per-acre of pesticide, applied within $500 \mathrm{~m}$ of each subject's residence annually for 1974-1999, was estimated. However, these data do not appear in the paper. Rather, each subject has been counted only as exposed or unexposed in either or both of the two 10- to 16-year time windows. It would seem that the available database would have allowed the researchers of this paper to describe each subject in terms of the number of years, and the specific years, of his/her exposure to each pesticide. This, in turn, would have allowed an assessment of dose-response and, possibly, even of induction periods. Such analyses may have been of considerable value in evaluating causality, especially for the younger subjects. Neither of these two variables is addressed in the paper. Separate analyses are presented for two time windows of exposure, 1974-1989 and 1990-1999 and for the entire period 1974-1999. The statement from the abstract regarding an odds ratio (OR) of 2.27 for 'either maneb or paraquat alone', relates to persons aged $\leq 60$ at diagnosis in the first, 1974-1989, time window. It seems in Table 3 of that study as pertaining to 'paraquat or maneb only' (presumably when compared with exposure to neither or to both). However, Table 2 of that study indicates that for both age groups in the entire 1974-1999 time window the OR for 'paraquat only' was 1.01 whereas that for 'maneb only' was 3.04. Data on paraquat alone are not provided for either of the two component time windows or for either specific age group. Therefore, the OR of 2.27 almost certainly reflects a putative association of PD with maneb, as that pesticide (in Table 2 of that study) has an OR of 3.04.

The analyses for both exposure time windows (and overall) suggest that among subjects $\geqslant 60$ years, the OR ranges from 4.17 to 5.74 for the exposure of 'both paraquat and maneb'. This is the basis for the interpretation in the abstract that the two pesticides together increase the risk of PD among younger people. It implies an interaction between maneb and paraquat in the causation of PD - surprising in view of the fact that paraquat alone seems to have virtually no association with PD. In Table 2 of that study, the OR for paraquat exposure only is 1.01 , for maneb only is 3.04 , but for 'both' it is 1.75 , which is not suggestive of any interaction between the two. Nevertheless, the findings in Table 3 of that study (subjects aged $\geqslant 60$ years) suggest that paraquat and maneb do interact. It is not clear why the results of this study are so different for the two age groups discussed; no doubt the 
Table 1 Summary results of studies considering the association between PQ and Parkinson's disease

\begin{tabular}{|c|c|c|c|c|}
\hline & Cases & Controls & & \\
\hline Study, year, country & Exposed/non-exposed & Exposed/non-exposed & Relative risk $(95 \% \mathrm{Cl})$ & Notes \\
\hline Hertzman et al., 1990, Canada & $4: 53$ & $0: 121$ & - & \\
\hline Hertzman et al., 1994, Canada & $6: 121$ & $4: 120$ (hospital) $5: 116$ (community) & $1.25(0.34-4.63) 1.11(0.32-3.87)$ & \\
\hline Seidler et al., 1996, Germany & $1: 379$ & $0: 755$ & - & \\
\hline Liou et al., 1997, Taiwan & $31: 89$ & $22: 218$ & $3.22(2.41-4.31)$ & \\
\hline Kuopio et al., 1999, Finland & $3: 120$ & $5: 239$ & $1.2(\mathrm{~ns})$ & \\
\hline Engel et al., 2001, USA & $20: 29$ & - & $0.8(0.5-1.3)$ & Parkinsonism \\
\hline Firestone et al., 2005, USA & $2: 154$ & $2: 239$ & $1.7(0.2-12.8)$ & \\
\hline Kamel et al., 2007, USA & $\begin{array}{l}14: 63 \\
11: 72\end{array}$ & $\begin{array}{l}11266: 44665 \\
7385: 48549\end{array}$ & $\begin{array}{l}1.8(1.0-3.4) \\
1.0(0.5-1.4)\end{array}$ & $\begin{array}{l}\text { Prevalent PD } \\
\text { Incident PD }\end{array}$ \\
\hline
\end{tabular}

younger patients were exposed at younger ages (from Table 1 it is possible to estimate their average year of birth as 1951) as they were only about 12 years old when paraquat was introduced. Even so, many of them were likely exposed only, or mainly, when older. In comparison, the average year of birth of the older cases was about 1931, and hence they were exposed to paraquat only at ages $\geqslant 30$ years. It is reasonable to consider that the only meaningful difference in age at exposure is likely to be that some young cases, but not older cases, were exposed while in their 20 s and fascinating to consider that an analysis of dose-response and induction (latent) period might be developed that might support or refute a causal relationship. Alternatively, it must be possible that the different procedures used for selecting younger and older controls produced the age-specific findings that are reported.

Ritz and her colleagues, ${ }^{22}$ in a case-control study of 324 incident cases of PD and 334 population controls in rural California, have reported that dopamine transporter (DAT) A clade diplotype and 3'VNTR 9/9 carriers are at increased risk for PD. The researchers further report that genetic susceptibility conferred by DAT A clade diplotype and 3'VNTR 9/9 interacts with joint exposure to paraquat and maneb to increase the risk for Parkinson's disease significantly. They used a 'population-based approach' although the nature of this is not clear. Certain cases were excluded because they were contacted more than 3 years after diagnosis and because they had 'other causes' of PD. The county-by-county distribution reported is different for cases and controls (see Table 1 in that study). Residence in Fresno County is strongly associated with PD, and these factors combined with the extremely low response rate may indicate that the case series and the controls are not representative of a defined population and may not be comparable.

The response rates were variable. For cases, the rate may be anywhere between 35 and $62 \%$, depending on which 'exclusions' are considered to be realistic. Exposure was determined in an unusual way, as it seems that 'high exposure' means exposure to 'paraquat and maneb', but it is not clear whether the so-called high exposure was either heavy or frequent. There are some apparent inconsistencies with the Costello et al. ${ }^{21}$ paper. The genetic findings were similar for younger (age $<61$ years) and older subjects. This seems difficult to reconcile with the report of Costello in which associations were different between younger and older subjects and there is a further problem in the use of 'zero' and 'low' exposures, combined, to designate the reference value for exposure to pesticide.

Other findings are difficult to interpret. For example, Table 3 of that study shows that the association between number of alleles and PD risk occurs at $3+$, not $2+$, alleles and thus it is unclear why $2+$ is used in Table 4 . Importantly, a $2 \times 2 \times 2$ interaction (paraquat, maneb and genetic susceptibility in at least dichotomous categories) is reported for 324 cases. Several thousand cases would indeed be required to document gene-environment interactions. ${ }^{23}$ It is established that most positive results in underpowered studies are false positives; ${ }^{24,25}$ indeed, this issue has forced those undertaking genetic association studies to set statistical significance limits at $P \leqslant 10^{-6}$.

The major finding of the study relating to gene-environment interaction is illustrated in Table 4 of that study, which shows that alleles in the $5^{\prime}$ and $3^{\prime}$ region of the DAT gene have little or no association with PD except in the presence of 'high' exposure (as defined, uniquely, in this study). This 'high' exposure is not associated with $P D(O R=0.88$ for zero alleles) except when $1+$ alleles are present. The researchers suggest that in the presence of 'high' pesticide exposure and one allele, the OR increases to 2.99. The presence of $2+$ alleles (most of the individuals with $2+$ alleles have 2 alleles and approximately $30 \%$ have $3+$ ) increases the OR to 4.53 (a true interaction would have suggested an OR $>6$ (additive) or $>9$ (multiplicative).

In conclusion, the evidence available from epidemiological studies is fragmentary and insufficient to establish whether herbicides and $P Q$ in particular increase the risk for $P D$. The overall epidemiological evidence from combined exposure studies and those limited to $P Q$ does not support the existence of a specific association between PQ and PD.

\section{PQ Toxicity in Animal Models and the Underlying Mechanisms}

Chemical properties and distribution. $P Q\left(1,1^{\prime}\right.$-dimethyl $4,4^{\prime}$-bipyridinium in its reduced form) is a member of a chemical class known as bipyridyl derivatives, which includes diquat (1,1'-ethylene-2,2'-bipyridylium dibromide) and cyperquat that has the same structure as the MPTP metabolite $\mathrm{MPP}^{+}$(see below). One-electron reduction of $P Q^{26-29}$ probably underlies its toxic effects in the lung 
after accidental ingestion. Reduced $P Q$ is then rapidly reoxidized to its cation form by molecular oxygen with the formation of superoxide radicals in a classical redox-cycling reaction.

Because $P Q$ is highly polar, it is poorly absorbed from the gastrointestinal tract. Over $50 \%$ of a single dose administered to rats localizes to the gut at some $32 \mathrm{~h}$ after administration ${ }^{30}$ and only approximately 5 to $10 \%$ of an ingested dose is absorbed, although the presence of emulsifiers and/or cosolvents may enhance absorption. Excretion occurs in urine and faeces with approximately $45 \%$ of a single dose being excreted after $48 \mathrm{~h}$ in rats. Because of these properties and some early evidence that $P Q$ is largely excluded from the brain, ${ }^{31}$ it has been assumed that $P Q$ would not cross the blood-brain barrier (BBB) to a significant extent. After a single administration, most of the $P Q$ that reaches the brain is apparently associated with structures outside the BBB (i.e., the pineal gland and the cerebral ventricles) or with three areas of the brain: the anterior portion of the olfactory bulb, the hypothalamus and the area postrema, which do not have a tight BBB. ${ }^{32}$

Shimizu and colleagues ${ }^{33}$ challenged these initial observations. Using a brain microdialysis technique in rats, they showed that $P Q$ could be found in the dialysate after subcutaneous administration. The researchers suggested that penetration of $P Q$ into the brain did not result from the destruction of $B B B$ function by $P Q$ or a $P Q$ radical, but suggested that $P Q$ is taken up into the brain through the neutral amino acid transport system and then transported into striatal cells. However, this study was carried out in young animals and the possibility exists that age-dependent changes in the BBB may be critical for the uptake of $P Q$ in brain. Several studies now agree that $P Q$ penetration into the brain is an age-dependent phenomenon. Higher PQ levels can be found in neonatal brains compared with brain regions of adult and elderly rats, ${ }^{32}$ or in either very young (2 weeks old) or very old (12 and 24 months old) animals when compared with normal adult (3-month-old rats). ${ }^{34,35}$ Thus, it is apparent that neonatal or very young animals are better able to sequester $P Q$. Because $P Q$ can cross the placental barrier, studies in which exposure occurs before birth or in general before the full development of a competent BBB are of little significance in unveiling the potential risk of developing PD after exposure to $P Q$.

Neurotoxicity of PQ, MPTP and other dopamine congeners. Several studies suggest that systemic administration of $P Q$ can cause neuronal damage and a parkinsonian-like syndrome in experimental animals (primarily $\mathrm{C} 57 \mathrm{BI} / 6$ mice or rats). The linking mechanism between PQ exposure and Parkinson's disease is suggested by the alleged chemical similarity between this compound and others known to cause a parkinsonian syndrome, particularly MPTP. MPTP can reproduce most of the biochemical, neuropathological and clinical characteristics of human parkinsonism in both human and non-human primates, ${ }^{36}$ with the notable exception of Lewy body formation. MPTP toxicity has been studied in cell systems in mice and in non-human primates. In rats, dopaminergic neurodegeneration is observed at high doses, ${ }^{37}$ whereas mice have become the most commonly used species for MPTP studies as they develop a dopaminergic degeneration that may be related to human parkinsonism.

The chemical similarity between MPTP/MPP ${ }^{+}$and PQ has been regarded as evidence for a similar cytotoxic mechanism and a common route of brain penetration. Nevertheless, the obvious difference in polarity between PQ and MPTP has implications for their ability to cross the BBB. Studies on brain distribution of $P Q$ in rodents have produced disparate results. The evidence can be summarized as follows: $P Q$ brain penetration is an age- and species-dependent process, with young mice ( $<8$ weeks) being more susceptible to $P Q$ brain accumulation. In contrast, there is no doubt that MPTP can cross the BBB and cause toxicity after being metabolized to $\mathrm{MPP}^{+} . \mathrm{MPP}^{+}$is non-neurotoxic when administered systemically. $P Q$ has two positive charges and thus different biological properties affecting both its transport across the BBB and its cellular uptake. PQ is not transported by the dopamine carrier - as believed initially - and does not affect dopamine uptake. . $^{38,39}$

The initial biochemical lesions produced by $\mathrm{PQ}$ and MPP ${ }^{+}$ differ substantially. The primary mechanism of MPP ${ }^{+}$toxicity is impairment of mitochondrial respiration by the inhibition of complex I of the electron transport chain. This may result in excess radical formation and subsequent oxidative/nitrosative stress. Unlike $\mathrm{MPP}^{+}, \mathrm{PQ}$ exerts its toxicity by cellular redox cycling, ${ }^{28,29,40}$ although a recent study in isolated mitochondria questions previous findings and indicates complex I as a target for $P Q .{ }^{41}$ In clear contrast with the latter study, recent work suggests that $P Q$ can still exert neurotoxic effects after a knock down of complex I. ${ }^{42}$ Notably, this study also suggests that complex I is not involved in MPP ${ }^{+}$toxicity, and therefore it concludes that complex I is not relevant in PD. The latter conclusion is arguable. It is necessary to realize that redox active agents may interact with different electron acceptor/ donors depending on the redox potential and the reducing environment. Thus, removing one or more electron acceptors/ donors may simply shift redox reaction to the next best biological partner. Although it is generally accepted that $P Q$ will cause cellular toxicity by oxidative stress, it is difficult to reach a definitive conclusion on $P Q$ major intracellular targets. Work in dopaminergic neurons has shown that the most effective redox cyclers also cause the highest degree of dopaminergic cell injury. ${ }^{28}$ Most notably, of three pesticides selected in this study, $P Q$ was the least toxic. Further evidence that oxidative stress is the predominant mechanism by which $P Q$ causes toxicity is the generation of 4-hydroxynonenals in vivo. ${ }^{27}$

It is possible to conclude from these studies that the mechanisms of toxicity of $P Q$ in neuronal cultures, cell lines and non-neuronal cells (lung, kidney and hepatocytes) are similar. It is clear that a chemical that is capable of triggering redox cycling/oxidative stress will do it regardless of the cellular system in which it operates in the presence of an adequate electron donor. The issue is therefore whether dopaminergic neurons and selective subpopulations of other brain cells will sequester sufficient $P Q$ to trigger a lethal oxidative stress. As indicated above, most studies in vitro will inevitably show this paradigm. Studies in vivo also seem to suggest this possibility. However, many studies in the 
literature are carried out in species that may have a high intrinsic sensitivity to neurotoxicants (C57/BL6) or in relatively young animals (ca 8-12 weeks old) in which $P Q$ uptake may be more significant. Caution should be exercised when deciding whether sensitivity to $P Q$ toxicity of a given strain may reflect $P Q$ penetration into the brain or rather an inbred trait for increased susceptibility. Because $P Q$ distribution in rats and mice may differ and as there are no age-related studies analysing the distribution and toxicity of $P Q$ in vivo in different species under the same experimental conditions, our knowledge of $P Q$ distribution in brain and its relation to neurotoxicity remains fragmentary. A schematic description of the reactions and CNS cells involved in cell death by $P Q$ and MPTP is presented in Figure 1.

Notably, gene expression patterns differ significantly after exposure to either $\mathrm{PQ}$ or MPP ${ }^{+43}$ Although both chemicals engage the ubiquitin-dependent protein catabolism to promote toxicity, overall their adverse effects are regulated by non-overlapping sets of conserved genes in yeast experiments. Notably, 80 and $76 \%$ of enriched yeast genes that show sensitivity to MPP ${ }^{+}$and $\mathrm{PQ}$ toxicity, respectively, have highly conserved homologues in the human genome, which suggest that similar studies in mammalian cells may reveal analogous differences. From these data, it seems plausible that although some downstream processes triggered by $P Q$ and $\mathrm{MPP}^{+}$are shared (the activation of the ubiquitin/ proteasome system) the initial steps that lead to the activation of this system may be different.

Thus, PQ and MPTP seem to engage different cytotoxic mechanisms. Despite studies in which $P Q$ is used to model redox cycling, ${ }^{41} \mathrm{PQ}$ is not an efficient inhibitor of mitochondrial complex I $\left(\mathrm{IC}_{50}\right.$ of $\left.7 \mathrm{mM}\right){ }^{39} \mathrm{~A}$ major difference in the interaction with cellular proteins between $P Q$ and complex I acting chemicals is also highlighted in a study carried out in $C$. elegans, in which the patterns of vulnerability and rescue have been compared after genetic manipulation. ${ }^{44}$ Ved et al. ${ }^{44}$ created a susceptibility model in $C$. elegans by overexpressing $\alpha$-synuclein (which is normally not expressed in the worm) and deleting Parkin or knocking down DJ-1. C. elegans lines with these transgenic traits were more vulnerable than non-transgenic worms to mitochondrial complex I inhibitors including Rotenone. However, the sensitivity to PQ did not increase.

In a study by Ramachandiran et al., ${ }^{38}$ it is shown that $P Q$ specifically oxidizes the cytosolic form of thyoredoxin and activates JNK and caspase 3. Conversely, $\mathrm{MPP}^{+}$and rotenone, which directly affect complex I activity, oxidize the mitochondrial form of thioredoxin without activating JNK or caspase 3 . In both cases, loading cells with exogenous dopamine does not exacerbate the toxicity of either compound. These data suggest that oxidative modification of cytosolic proteins is important for $P Q$ toxicity, whereas inactivation or oxidation of mitochondrial proteins is more important for $\mathrm{MPP}^{+}$and rotenone toxicity. In addition, intracellular dopamine does not seem to exacerbate the toxicity of these dopaminergic neurotoxicants, at least in this model.

It is apparent from this analysis that the initial targets and toxicity mechanisms of $\mathrm{PQ}$ and $\mathrm{MPP}^{+}$differ (although all may share an oxidative component).

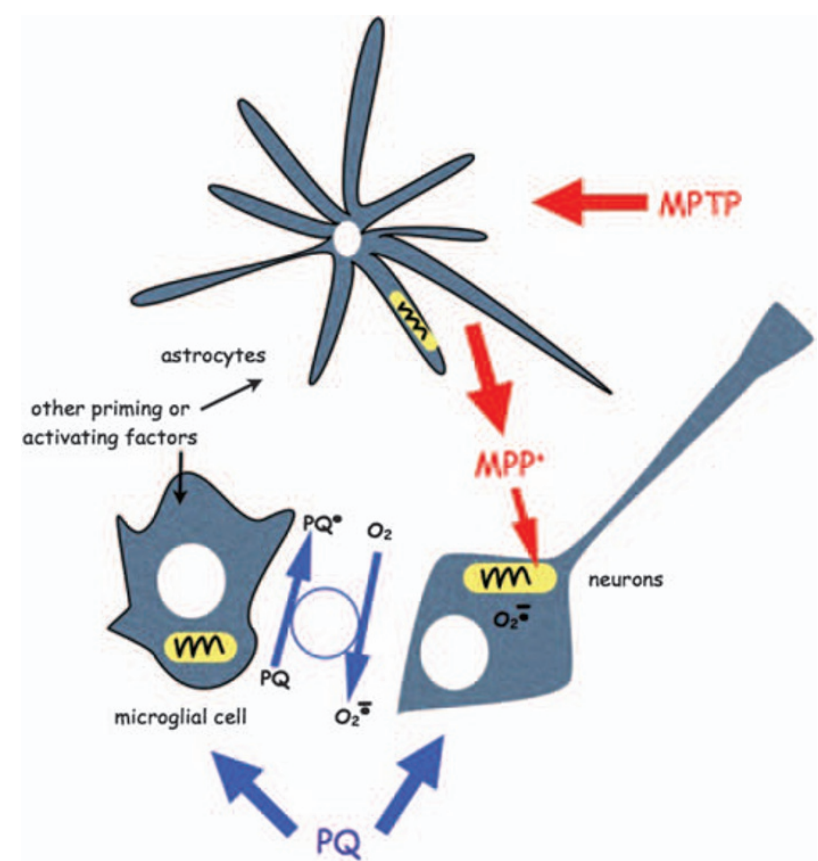

Figure 1 Mechanisms of $P Q$ and MPTP toxicity. $P Q$ can cause an oxidative stress either intracellularly by redox cycling or by activation at cell surfaces by the NADPH oxidase (see text). Mitochondria can be affected indirectly or directly by PQ. In neurons the effects of $P Q$ are believed to be primarily cytosolic. MPTP is converted to its toxic metabolite $\mathrm{MPP}^{+}$and then sequestered through the dopamine transporter in dopaminergic neurons in which it primarily affects complex I, promoting oxidative stress and mitochondrial damage

Modelling PD in rodents and simple organisms. Impaired mitochondrial function is a predominant feature in cases of Parkinson's' disease due to genetic or environmental modifications, which result in 'mitochondrial stress'. This may directly compromise the neuron or cause alterations in neurotransmitter release, resulting in post-synaptic damage. A pro-inflammatory component is increasingly considered to be important in the pathogenesis of several neurodegenerative conditions including $\mathrm{PD},{ }^{45}$ and pro-inflammatory activation of glial cells may be pivotal in disease onset. ${ }^{46,47}$

Although many of the genes involved in PD have been identified, their interactions are still unclear. Mutations of $\alpha$-synuclein or duplications of $\alpha$-synuclein are linked to familial PD and $\alpha$-synuclein is a component of Lewy bodies. $\alpha$-synuclein tends to form intracellular fibrils and aggregates, particularly after oxidative stress. Loss of an ubiquitin ligase, Parkin, is responsible for autosomal recessive juvenile parkinsonism. Finally, mutations in genes coding for $\mathrm{UCH}-$ $\mathrm{L} 1$ and Pink-1 are linked to autosomal recessive PD, whereas mutations in LRRK2 are linked with autosomal dominant PD. Recent work has shown that mutations in the mitochondrial protease $\mathrm{HtrA}^{48}$ are also linked to $\mathrm{PD}$ downstream of the kinase, Pink $1 .^{49}$ Mutations in Parkin are recognized as the most common cause of familial parkinsonism and may be involved in sporadic PD. Parkin seems to work as a broadspectrum neuroprotectant, the efficacy of which decreases with ageing. ${ }^{50}$ 
With a better understanding of the genetic alterations involved in familial PD, several transgenic mouse models have been generated that can reproduce many features of parkinsonism. Animal models generated by mutations or deletions of the relevant Parkinson-related genes have also been used to examine the effects of $P Q$. Manning-Bog et $a l^{51,52}$ have shown that $P Q$ can cause $\alpha$-synuclein aggregation in wt C57BI/6 mice, whereas overexpression of $\alpha$-synuclein protects against herbicide toxicity. $\mathrm{MPP}^{+}$, rotenone and $P Q$ have all been shown to alter Parkin solubility, which facilitates its aggregation, decreases proteasome activity and causes cell injury and death. ${ }^{53}$ In contrast, in the C. elegans controlled genetic background, the sensitivity model proposed by Ved et al. ${ }^{44}$ has shown that $P Q$ toxicity is not enhanced by the Parkinson-linked genetic modifications, at variance with chemicals exerting an effect specifically on mitochondrial complex I.

These observations do not resolve the question of whether $P Q$ exposure can realistically facilitate the onset of $P D$ in humans. The additional stress triggered by any redox-cycling agent in genetically susceptible animal models may indeed prompt the development of pathological features. In line with this assumption are observations that $P Q$ increases fibrillar aggregation of $\alpha$-synuclein. ${ }^{54}$ Notably, the protection by $\alpha$-synuclein overexpression is independent of $P Q$-induced formation of synuclein aggregates. ${ }^{51}$

There are several intrinsic limitations in modelling PD by chemical or genetic manipulation. The major controversy about the use of chemically induced models of PD has been related to the lack of certain critical features of human parkinsonism and therefore the potential value of the model systems to study neuroprotective therapeutics. For example, rodent exposure to 6-hydroxydopamine has been used extensively to model damage in catecholaminergic neurons and induce dopaminergic neuronal death, but it neither shows the formation of cytoplasmic inclusion (Lewy bodies) nor involves other brain areas normally affected in PD. Reports of parkinsonian-like tremor are very rare in studies of 6-hydroxydopamine-injured rodents and only occasionally have akinesia and rigidity been described. Finally, the mechanism by which 6-hydroxydopamine can kill neurons does not necessarily reflect the mechanism that is involved in spontaneous PD in humans. In MPTP-treated animals a number of symptoms replicate PD and MPTP exposure has been shown to cause parkinsonism in humans. However, the slow progression of the idiopathic human disease, the clinical symptoms of the movement disorder and the classical Lewy body formation are not reproduced. In general, the animal models of PD, including toxin-induced models, fulfil most of the required features, but not all of them, and therefore cannot be taken as bona fide models for PD. This is recognized widely in the field and research progresses towards models that recapitulate more closely the features of human parkinsonism. These should include: (1) a normal set of nigral-dopaminergic neurons at birth followed by a selective gradual loss of these cells beginning in adulthood; (2) easily detectable and quantifiable model deficit, for example, akinesia, rigidity and tremor; and (3) formation of Lewy bodies.
Multiple hits: Rodent Studies and the Hypothesis that Multiple Triggers cause Neurodegeneration

Perinatal exposure: $P Q$ as a sensitizer. Studies primarily from the Cory-Slechta group ${ }^{55-57}$ have suggested that exposure to $P Q$ and maneb (a dithiocarbamate pesticide) during the prenatal and perinatal period can either cause a reduction in the number of dopamine neurons directly, or cause an increased susceptibility to degeneration of these neurons after subsequent environmental insults or as a result of ageing. It is interesting that exposure to the proinflammatory agent LPS can sensitize neurons in a similar manner. This would suggest that sensitizing environmental factors and/or inflammatory conditions in the perinatal period might favour the early onset of parkinsonism. It is conceivable that any pro-oxidant that can cross the BBB and the placental barrier may interfere with normal neurodevelopment with delayed consequences. However, although $P Q$ may cause developmental or post-developmental effects in rodents, the relevance of such studies to help understanding $P Q$ neurotoxicity in humans is limited. Although it is plausible that $P Q$ may cross the placental barrier, it is unclear to what extent. In addition, there is no evidence to date that: (1) environmental exposure of pregnant women is significant; and (2) $P Q$ gestational exposure is linked to early-onset PD in humans. Finally, there is no convincing evidence that perinatal inflammation - which is far more common than $\mathrm{PQ}$ exposure - increases risk for PD. In support of this hypothesis, there is a single additional study. Gravid female rats were injected intraperitoneally with LPS at embryonic day $10.5 .^{58}$ Pups showed reduced numbers of tyrosine hydroxylase positive neurons, an effect that was still present at 33 days after a single injection of LPS.

Thus, perinatal rodent exposure models may help to unveil biological interactions, but are obviously inappropriate as models of human exposure and its consequences.

The pro-inflammatory or 'multiple hits' hypothesis. Recent work by Purisai et al. ${ }^{59}$ has shown that administration of three weekly injections of $P Q$ causes a significant loss of the dopaminergic neurons in the substantia nigra, pars compacta. Along with these findings is the observation that treatment with $P Q$ increases the levels of $\alpha$-synuclein. In this paradigm, a single PQ injection did not produce toxic effects, whereas subsequent injections triggered oxidative injury and neurotoxicity. $P Q$ accumulation may explain this finding. However, repeated injections of $P Q$ would be expected to produce a progressive pattern of neurodegeneration. Instead, after three different exposures, only the second $P Q$ treatment caused a significant loss of nigral striatal neurons. Another explanation may be that the initial exposure can exert an effect as a priming event, enhancing neuronal vulnerability to a subsequent toxic insult. This 'multiple hit' hypothesis implies that neurodegeneration would begin shortly after two $P Q$ injections and would progress over the following few days. Once the maximum effect is reached after the second exposure, no further cell loss occurs. According to this paradigm, the first injection would sensitize brain cells in a non-lethal way whereas the second injection would trigger 
irreversible injury. Purisai et al. ${ }^{59}$ suggest that neurotoxicity of $P Q$, as well as other pro-oxidants that indeed cross the $\mathrm{BBB}$, may be due to a two-tiered effect, priming (perhaps of microglia) and a secondary injury to the neurons because of the combination of a pro-inflammatory response and a direct neuronal oxidative stress. The major source of oxidative stress may in fact, be the resident microglia. In Purisai et al. ${ }^{59}$ studies there is evidence that a single $P Q$ dose is sufficient to increase the number of cells with immunohistochemical, morphological and biochemical characteristics of activated microglia, including the induction of NADPH oxidase. The anti-inflammatory drug, myocyclin, blocks this microglial response and under these circumstances, $P Q$ fails to cause both oxidative stress and neurodegeneration in subsequent exposures. If microglial activation is induced by pre-treatment with lipopolisaccaride (LPS), a single $P Q$ exposure becomes capable of triggering loss of dopaminergic neurons. A final experiment using mutant mice that lack functional NADPH oxidase shows that these animals were spared from neurodegeneration caused by repeated $P Q$ exposure. These data therefore suggest that microglial activation and the consequent induction of NADPH oxidase may exert an effect as risk factors for the dopaminergic neuronal loss in rodents. It is unclear whether this paradigm applies to human parkinsonism. In addition, given the frequency of pro-inflammatory events in life, one may ask what would be the role of a rather infrequent exposure to $P Q$ in comparison.

Nevertheless, this working hypothesis fits with the general idea that neurodegenerative conditions may be caused by a series of unrelated events. Priming may include genetic factors, which would make certain neuronal populations or individuals more sensitive to subsequent environmental factors. The latter may be triggers of inflammation or exposure to toxic compounds. The reason why dopaminergic neurons may be more sensitive to oxidative stress can be explained by a number of findings: expression levels of stress-related proteins $^{49}$ or an increased sensitivity of mitochondria to oxidative stress. This would explain why compounds, such as $6-\mathrm{OH}$-dopamine, MPP + or $\mathrm{PQ}$ with apparently distinct cytotoxic triggers, can promote a parkinsonian syndrome if injected systemically. However, because LPS sensitizes to other unrelated forms of brain injury (e.g., tau pathology), ${ }^{60}$ and because the sensitization is carried out with an LPS pretreatment protocol, it may be likely that these experiments only reveal an intrinsic weakness of the animal model. Although there is increasing evidence that pro-inflammatory conditions can facilitate onset of neurodegeneration, there is no firm evidence that even repetitive pro-inflammatory events in humans are linked to PD.

Cell death mechanisms and neurodegeneration. Although transgenic animal models are becoming more and more sophisticated, rodent models for neurodegenerative diseases, in particular Alzheimer's and Parkinson's diseases, are still inadequate. Neither $P Q$ nor other chemical agents do indeed model human PD. The complex and slow progressive pathology in humans is hardly mimicked by the rapid disruption of nigro-striatal neurons in the rodent chemical models. Despite this obvious problem, chemicals have long been used to mimic neurodegenerative conditions in the brain. Of these, some are used to induce pharmacological lesions, such as 6-hydroxydopamine, but obviously there is no relation with human exposure. Others such as MPTP have become widely used tools because of cases of human toxicity. Finally, PQ has become a tool for the alleged chemical similarity with MPTP because of being a widely used agent in agriculture, but to date there is no evidence that links it to parkinsonism in humans. In fact, none of the chemicals above produce the progressive lesions that characterize neurodegenerative diseases. The latter develop in humans over decades and most likely do not result from a rapid neuronal death as that caused by chemicals. There is mounting evidence that loss of synaptic connectivity, axonal and dendritic damage take place long before cell death mechanisms are activated. The latter being apoptotic or necrotic seem to be initially restricted to neurons. Many pathways are now believed to converge on mitochondria. Protein misfolding, disorders in axonal trafficking, and vesicle recycling or genetic mutations in the nuclear or mitochondrial genomes can compromise mitochondrial function and lead to loss of synapses and/or neuronal death (Figure 2). The chemicals discussed in this study can directly or indirectly affect mitochondrial function and therefore cause neurodegeneration in cultured neurons, in animal models or, in some cases, in humans. Any mitochondrial toxin that would be experimentally forced to gain access to neurons in high-enough concentrations would likely cause a similar damage, without necessarily posing a real risk to human health. Therefore, although informative, chemical models have little relevance in the understanding of how neurodegenerative diseases develop and progressively damage the nervous system.

\section{Conclusions}

The scientific process leading to identification of human risk is complex and involves both epidemiological and experimental data. Epidemiological or clinical studies may identify associations between a given pathology and known risk factors, but risk evaluation is more difficult when epidemiological and clinical data are lacking or incomplete. In such cases, animal experimentation is essential to establish whether or not there is an increased risk. Identification of effects in animals, and understanding the targets and mechanism of action, pharmacokinetics and tissue distribution are essential in the determination of risk.

The epidemiological and clinical evidence that $P Q$ may favour the onset of PD is inconclusive. The experimental studies that might inform us do not reflect human exposure, they are performed in selected strains (which may show increased sensitivity) and they reveal an increased sensitivity to oxidative damage of a subset of neurons, regardless of the pro-oxidant used. They offer a plausible hypothesis of how $P Q$ may exert an effect on rodents, but provide little or no help with risk prediction in humans.

Public and often scientific perception of risk is based on extrapolation and approximation. The precautionary principle is the bane of a truly scientific approach, but one behind which we comfortably hide when we do not know better. In the case 
13. Liou HH, Tsai MC, Chen CJ, Jeng JS, Chang YC, Chen SY et al. Environmental risk factors and Parkinson's disease: a case-control study in Taiwan. Neurology 1997; 48 1583-1588.

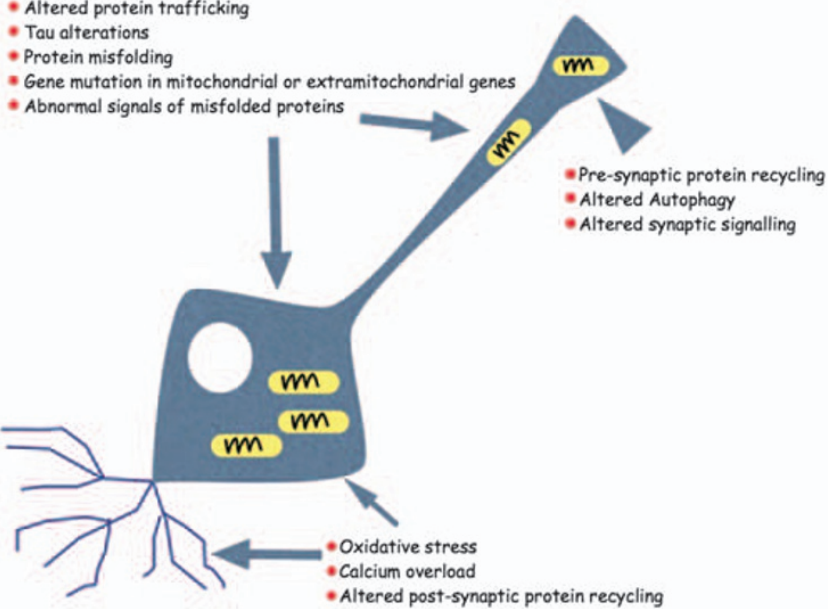

Figure 2 Possible mechanisms leading to synaptic, dendritic and axonal damage in neurodegeneration

of $P Q$, the link between experiments in rodents and potential human adverse effects is weak. Nevertheless, it is likely that the work on animals linked to low-stringency epidemiological evidence will create concern. To definitely answer the question of $P Q$ toxicity in humans, better epidemiological studies and thorough studies in primates will be required.

\section{Conflict of interest}

Over the past several years, the researchers have worked with pharmaceutical and chemical companies as external advisors. This work reflects their scientific experience and independent views.

1. Warner TT, Schapira AH. Genetic and environmental factors in the cause of Parkinson's disease. Ann Neurol 2003; 53 (Suppl 3): S16-S23; discussion S23-25.

2. Schapira AH. Neurobiology and treatment of Parkinson's disease. Trends Pharmacol Sci 2009; 30: 41-47

3. Litvan I, Bhatia KP, Burn DJ, Goetz CG, Lang AE, McKeith I et al. Movement Disorders Society Scientific Issues Committee report: SIC Task Force appraisal of clinical diagnostic criteria for Parkinsonian disorders. Mov Disord 2003; 18: 467-486.

4. Barbeau A. Manganese and extrapypamidal disorders (a critical review and tribute to Dr. Georg C. Cotzias). Neurotoxicology 1984; 5: 13-35.

5. Li AA, Mink PJ, Mclntosh LJ, Teta MJ, Finley B. Evaluation of epidemiologic and anima data associating pesticides with Parkinson's disease. J Occup Environ Med 2005; 47: 1059-1087.

6. Brown TP, Rumsby PC, Capleton AC, Rushton L, Levy LS. Pesticides and Parkinson's disease-is there a link? Environ Health Perspect 2006; 114: 156-164.

7. Engel LS, Checkoway H, Keifer MC, Seixas NS, Longstreth Jr WT, Scott KC et al. Parkinsonism and occupational exposure to pesticides. Occup Environ Med 2001; 58: 582-589.

8. Baldi I, Lebailly P, Mohammed-Brahim B, Letenneur L, Dartigues JF, Brochard P. Neurodegenerative diseases and exposure to pesticides in the elderly. Am J Epidemiol 2003; 157: 409-414.

9. Frigerio R, Sanft KR, Grossardt BR, Peterson BJ, Elbaz A, Bower JH et al. Chemical exposures and Parkinson's disease: a population-based case-control study. Mov Disord 2006; 21: 1688-1692.

10. Ascherio A, Chen H, Weisskopf MG, O'Reilly E, McCullough ML, Calle EE et al. Pesticide exposure and risk for Parkinson's disease. Ann Neurol 2006; 60: 197-203.

11. Dick FD, De Palma G, Ahmadi A, Scott NW, Prescott GJ, Bennett J et al. Environmental risk factors for Parkinson's disease and parkinsonism: the Geoparkinson study. Occup Environ Med 2007; 64: 666-672.

12. Seidler A, Hellenbrand W, Robra BP, Vieregge P, Nischan $\mathrm{P}$, Joerg $\mathrm{J}$ et al. Possible environmental, occupational, and other etiologic factors for Parkinson's disease: a casecontrol study in Germany. Neurology 1996; 46: 1275-1284.
14. Kuopio AM, Marttila RJ, Helenius H, Rinne UK. Environmental risk factors in Parkinson's disease. Mov Disord 1999; 14: 928-939.

15. Firestone JA, Smith-Weller T, Franklin G, Swanson P, Longstreth Jr WT, Checkoway H. Pesticides and risk of Parkinson disease: a population-based case-control study. Arch Neurol 2005; 62: 91-95.

16. Kamel F, Tanner C, Umbach D, Hoppin J, Alavanja M, Blair A et al. Pesticide exposure and self-reported Parkinson's disease in the agricultural health study. Am J Epidemiol 2007; 165: 364-374.

17. Hertzman C, Wiens M, Bowering D, Snow B, Calne D. Parkinson's disease: a case-control study of occupational and environmental risk factors. Am J Ind Med 1990; 17: 349-355.

18. Hertzman C, Wiens M, Snow B, Kelly S, Calne D. A case-control study of Parkinson's disease in a horticultural region of British Columbia. Mov Disord 1994; 9: 69-75.

19. Liou HH, Chen RC, Chen THH, Tsai YF, Tsai MC. Attenuation of paraquat-induced dopaminergic toxicity on the substantia nigra by (-)-deprenyl in vivo. Toxicol Appl Pharmacol 2001; 172: 37-43.

20. Tanner CM, Ross GW, Jewell SA, Hauser RA, Jankovic J, Factor SA et al. Occupation and risk of parkinsonism: a multicenter case-control study. Arch Neurol 2009; 66: 1106-1113.

21. Costello S, Cockburn M, Bronstein J, Zhang X, Ritz B. Parkinson's disease and residential exposure to maneb and paraquat from agricultural applications in the central valley of California. Am J Epidemiol 2009; 169: 919-926.

22. Ritz BR, Manthripragada AD, Costello S, Lincoln SJ, Farrer MJ, Cockburn M et al. Dopamine transporter genetic variants and pesticides in Parkinson's disease. Environ Health Perspect 2009; 117: 964-969.

23. Ioannidis JP, Trikalinos TA, Khoury MJ. Implications of small effect sizes of individual genetic variants on the design and interpretation of genetic association studies of complex diseases. Am J Epidemiol 2006; 164: 609-614

24. Peto R, Pike MC, Armitage P, Breslow NE, Cox DR, Howard SV et al. Design and analysis of randomized clinical trials requiring prolonged observation of each patient. I. Introduction and design. Br J Cancer 1976; 34: 585-612.

25. Peto R, Pike MC, Armitage P, Breslow NE, Cox DR, Howard SV et al. Design and analysis of randomized clinical trials requiring prolonged observation of each patient. II. Analysis and examples. Br J Cancer 1977; 35: 1-39.

26. McCarthy S, Somayajulu M, Sikorska M, Borowy-Borowski H, Pandey S. Paraquat induces oxidative stress and neuronal cell death; neuroprotection by water-soluble coenzyme Q(10). Toxicol Appl Pharmacol 2004; 201: 21-31.

27. McCormack AL, Atienza JG, Johnston LC, Andersen JK, Vu S, Di Monte DA. Role of oxidative stress in paraquat-induced dopaminergic cell degeneration. J Neurochem 2005 93: 1030-1037.

28. Bonneh-Barkay D, Langston WJ, Di Monte DA. Toxicity of redox cycling pesticides in primary mesencephalic cultures. Antioxid Redox Signal 2005; 7: 649-653.

29. Bonneh-Barkay D, Reaney SH, Langston WJ, Di Monte DA. Redox cycling of the herbicide paraquat in microglial cultures. Mol Brain Res 2005; 134: 52-56.

30. Murray RE, Gibson JE. Paraquat disposition in rats, guinea pigs and monkeys. Toxicol Appl Pharmacol 1974; 27: 283-291.

31. Widdowson PS, Farnworth MJ, Upton R, Simpson MG. No changes in behaviour, nigrostriatal system neurochemistry or neuronal cell death following toxic multiple oral paraquat administration to rats. Hum Exp Toxicol 1996; 15: 583-591.

32. Naylor JL, Widdowson PS, Simpson MG, Farnworth M, Ellis MK, Lock EA. Further evidence that the blood-brain-barrier impedes paraquat entry into the brain. Hum Exp Toxicol 1995; 14: 587-594

33. Shimizu K, Ohtaki K, Matsubara K, Aoyama K, Uezono T, Saito O et al. Carrier-mediated processes in blood-brain barrier penetration and neural uptake of paraquat. Brain Res 2001; 906: 135-142

34. Corasaniti MT, Strongoli MC, Pisanelli A, Bruno P, Rotiroti D, Nappi G et al. Distribution of paraquat into the brain after its systemic injection in rats. Funct Neurol 1992; 7: 51-56.

35. Corasaniti MT, Defilippo R, Rodino P, Nappi G, Nistico G. Evidence that paraquat is able to cross the blood-brain barrier to a different extent in rats of various age. Funct Neurol 1991; 6: $385-391$.

36. Langston JW, Ballard P, Tetrud JW, Irwin I. Chronic Parkinsonism in humans due to a product of meperidine-analog synthesis. Science (New York, NY) 1983; 219: 979-980.

37. Ossowska K, Smialowska M, Kuter K, Wieronska J, Zieba B, Wardas J et al. Degeneration of dopaminergic mesocortical neurons and activation of compensatory processes induced by a long-term paraquat administration in rats: Implications for Parkinson's disease. Neuroscience 2006; 141: 2155-2165

38. Ramachandiran S, Hansen JM, Jones DP, Richardson JR, Miller GW. Divergent mechanisms of paraquat, MPP+, and rotenone toxicity: oxidation of thioredoxin and caspase-3 activation. Toxicol Sci 2007; 95: 163-171.

39. Richardson JR, Quan Y, Sherer TB, Greenamyre JT, Miller GW. Paraquat neurotoxicity is distinct from that of MPTP and rotenone. Toxicol Sci 2005; 88: 193-201.

40. McCormack AL, Atienza JG, Langston JW, Di Monte DA. Decreased susceptibility to oxidative stress underlies the resistance of specific dopaminergic cell populations to paraquat-induced degeneration. Neuroscience 2006; 141: 929-937.

41. Cocheme HM, Murphy MP. Complex I is the major site of mitochondrial superoxide production by paraquat. J Biol Chem 2008; 283: 1786-1798. 
42. Choi WS, Kruse SE, Palmiter RD, Xia Z. Mitochondrial complex I inhibition is not required for dopaminergic neuron death induced by rotenone, MPP+, or paraquat. Proc Natl Acad Sci USA 2008; 105: 15136-15141.

43. Doostzadeh J, Davis RW, Giaever GN, Nislow C, Langston JW. Chemical genomic profiling for identifying intracellular targets of toxicants producing Parkinson's disease. Toxicol Sci 2007; 95: 182-187.

44. Ved R, Saha S, Westlund B, Perier C, Burnam L, Sluder A et al. Similar patterns of mitochondrial vulnerability and rescue induced by genetic modification of alpha-synuclein parkin, and DJ-1 in Caenorhabditis elegans. J Biol Chem 2005; 280: 42655-42668.

45. Schapira AH. Neuroprotection and dopamine agonists. Neurology 2002; 58 (4 Suppl 1): S9-18.

46. Liu B. Modulation of microglial pro-inflammatory and neurotoxic activity for the treatment of Parkinson's disease. AAPS J 2006; 8: E606-E621.

47. Wu XF, Block ML, Zhang W, Qin L, Wilson B, Zhang WQ et al. The role of microglia in paraquat-induced dopaminergic neurotoxicity. Antioxid Redox Signal 2005; 7: 654-661.

48. Strauss KM, Martins LM, Plun-Favreau H, Marx FP, Kautzmann S, Berg D et al. Loss of function mutations in the gene encoding Omi/HtrA2 in Parkinson's disease. Hum Mol Genet 2005; 14: 2099-2111.

49. Plun-Favreau H, Klupsch K, Moisoi N, Gandhi S, Kjaer S, Frith D et al. The mitochondrial protease HtrA2 is regulated by Parkinson's disease-associated kinase PINK1. Nat Cell Bio 2007: 1243-1252.

50. Thomas B, Beal MF. Parkinson's disease. Human Mol Genet 2007; 16 (Spec No 2): R183-R194.

51. Manning-Bog AB, McCormack AL, Li J, Uversky VN, Fink AL, Di Monte DA. The herbicide paraquat causes up-regulation and aggregation of alpha-synuclein in mice - Paraquat and alpha-synuclein. J Biol Chem 2002; 277: 1641-1644.
52. Manning-Bog AB, McCormack AL, Purisai MG, Bolin LM, Di Monte DA. alpha-synuclein overexpression protects against paraquat-induced neurodegeneration. J Neurosci 2003; 23: 3095-3099.

53. Wang C, Ko HS, Thomas B, Tsang F, Chew KC, Tay SP et al. Stress-induced alterations in parkin solubility promote parkin aggregation and compromise parkin's protective function. Human Mol Genet 2005; 14: 3885-3897.

54. Uversky VN, Li J, Fink AL. Pesticides directly accelerate the rate of alpha-synuclein fibril formation: a possible factor in Parkinson's disease. FEBS Lett 2001; 500: 105-108.

55. Cory-Slechta DA, Thiruchelvam M, Barlow BK, Richfield EK. Developmental pesticide models of the Parkinson disease phenotype. Environ Health Perspect 2005; 113: 1263-1270.

56. Barlow BK, Richfield EK, Cory-Slechta DA, Thiruchelvam M. A fetal risk factor for Parkinson's disease. Dev Neurosci 2004; 26: 11-23.

57. Thiruchelvam M, Richfield EK, Goodman BM, Baggs RB, Cory-Slechta DA. Developmental exposure to the pesticides paraquat and maneb and the Parkinson's disease phenotype. Neurotoxicology 2002; 23: 621-633.

58. Ling Z, Gayle DA, Ma SY, Lipton JW, Tong CW, Hong JS et al. In utero bacterial endotoxin exposure causes loss of tyrosine hydroxylase neurons in the postnatal rat midbrain. Mov Disord 2002; 17: 116-124.

59. Purisai MG, McCormack AL, Cumine S, Li J, Isla MZ, Di Monte DA. Microglial activation as a priming event leading to paraquat-induced dopaminergic cell degeneration. Neurobiol Dis 2007; 25: 392-400.

60. Kitazawa M, Oddo S, Yamasaki TR, Green KN, LaFerla FM. Lipopolysaccharideinduced inflammation exacerbates tau pathology by a cyclin-dependent kinase 5-mediated pathway in a transgenic model of Alzheimer's disease. J Neurosci 2005; 25 : 8843-8853. 